\title{
LINEAR ACCELERATION EMISSION: A DETAILED ANALYSIS
}

\author{
E. T. Rowe \\ University of Sydney
}

\begin{abstract}
An exact expression is given for the orbit of a charged particle accelerated by a non-propagating electrostatic wave. The corresponding current is obtained, for untrapped particles, as an expansion in Bessel functions, and a Lorentz transform allows us to treat the case of a propagating wave. An appropriate form for the absorption coefficient is derived, and an interesting angular and frequency dependence is revealed when the propagating wave is superluminal. Finally, the possible application to pulsars, particularly in explaining the multicomponent pulse profiles, is discussed briefly.
\end{abstract}

\section{Introduction}

The purpose of this paper is to explore linear acceleration emission by charged particles, accelerated by an electrostatic wave, with the electric field being treated exactly. In discussing application of the results to pulsars, we concentrate on waves with phase speed greater than the speed of light. The motivations for this work are as follows:

i. as*a process in its own right, linear acceleration emission (in an electrostatic wave) has not been treated in detail and in particular, no-one has considered the case of superluminal waves;

ii. a coherent emission mechanism capable of explaining the high brightness temperatures of radio pulsar emission has yet to be found, at least from the theoretical viewpoint and;

iii. there are general theoretical grounds for believing that the linear acceleration process will be present in pulsar magnetospheres.

Linear acceleration emission has to date been treated using a perturbation approach (e.g. Melrose 1978) in which the second order equation of motion is expanded in an arbitrary, small field. This approach does not permit identification of the constant of the particle motion and, being based on an iterative scheme, cannot sensibly be applied above the first order. An alternative, non-iterative expansion method (Rowe 1990) which can be applied to a monochromatic wave in the rest frame of the wave, involves expanding the Hamiltonian rather than the second order equation. This allows determination of the constant of the particle motion in an exact, open form when carried to all powers in the field. An exact treatment of acceleration by transverse waves was given by Gunn and Ostriker (1971), and here we perform the equivalent calculation for the electrostatic case to give a closed form for the orbit and drift velocity. This solution is valid for untrapped particles, with only minor modifications necessary for trapped particles. From the particle orbit we derive an expansion for the particle current, which we then Lorentz transform in order to obtain the result for an arbitrary wave with non-zero frequency, $\Omega$ [the work of Melrose (1978) was restricted solely to a time varying field-effectively a wave with infinite phase speed-and one finds that this obscures an interesting angular dependence of the resulting maser]. Krishnan and Sivaram (1983) also attempted to generalize to an arbitrary wave, within the iterative perturbation approach, however their calculations contained an error. Our calculation of the absorption coefficient follows Melrose (1980, p.157). We find that for a one-dimensional distribution the sign of the absorption is dependent on two factors:

i. the sign of the slope of the particle distribution and;

ii. the sign of the change in the particle momentum on emission (for superluminal waves this is not always positive when emission is in the forward direction).

Emission from plasmas with superluminal longitudinal oscillations has been considered before but not, to the author's knowledge, in connection with radio pulsars. It is well known that a relativistic electron-positron or electron-ion plasma wiil support superluminal oscillations (e.g. Silin 1960, Buti 1962, Imre 1962). More importantly, in a relativistic plasma, subluminal oscillations are very effectively Landau damped while the superluminal ones cannot be Landau damped. The linear acceleration process will, 
however, damp and/or amplify such waves and so, if they can be generated, it is important to understand how they may be converted into observable transverse radiation. In attempts to understand pulsar radio emission, the most widely invoked mechanism is curvature emission (e.g. Radhakrishnan 1969, Sturrock 1971, Gil 1986). Nevertheless, it is difficult to understand how the coherence of the radiation is attained for it is known that curvature radiation does not admit maser action in vacuo (Blandford 1975) nor in a plasma (Melrose 1978), and the most widely adopted solution, bunching (e.g. Sturrock, Petrosian and Turk 1975, Elsässer and Kirk 1976), is still theoretically unsatisfactory (e.g. Melrose 1981). Even if curvature emission is accepted, then there appears to be some other mechanism at work in the core of the polar cap where the emission characteristics are different from those of the hollow cone (e.g. Rankin 1983a). The mechanism considered here provides a possible alternative to curvature emission, or at least an additive, and the following points suggest it deserves attention:

i. in polar gap models some form of discharge across a spark gap is employed (e.g. Ruderman and Sutherland 1975) which could conceivably generate large amplitude plasma oscillations;

ii. the configuration of the pulsar magnetosphere is still poorly understood and;

iii. the linear acceleration mechanism allows masering. The mechanism is favored in a region of relatively straight, open or closed, field lines. Such a region of straight field lines would be severely constrained geometrically, confining the emission to a region near to the poles and close to the surface of the star.

This paper is organized as follows: Sections below give a) An exact solution for the orbit of untrapped particles, $b$ ) A derivation of the single particle current, $c$ ) An extension of the results to propagating waves, d) A calculation of the absorption coefficient for the emitted waves, and finally, a discussion of how the results apply to pulsars. In this paper we use units with $\hbar=c=1$ and $\mu_{0}=4 \pi$.

\section{Particle orbit: An exact solution}

Starting with the particle Hamiltonian for parallel wave, particle motion along the magnetic field

$$
m \gamma+\left(q E_{0} / K\right) \cos \left(K\left[z-z_{0}\right]\right)=m \gamma_{0},
$$

in the rest frame of the wave, where $E_{0}$ and $K$ are the electric amplitude and wavenumber of the wave, $m, q$ and $\gamma$ are the mass, charge and $\gamma$ factor of the particle and $z_{0}$ is an arbitrary origin, we find that particles are untrapped if $\gamma_{0}-1>\left|q \phi_{0}\right| / m$. We now integrate eq.(1) in the untrapped case to obtain the particle orbit in the unusual form of $t$ as a function of $z$ (it is impossible to invert analytically the resulting expression). We define the following parameters

$$
\begin{aligned}
r & =q E_{0} /(m K), \\
\xi_{-} & =v_{0}^{2} \gamma_{0}^{2}-r^{2}, \\
\xi_{+} & =v_{0}^{2} \gamma_{0}^{2}+r^{2}, \\
\Delta & =\sqrt{\left(\left(\gamma_{0}-1\right)^{2}-r^{2}\right)\left(\left(\gamma_{0}+1\right)^{2}-r^{2}\right)}, \\
\eta & =\frac{1}{\eta_{0}} \frac{\xi_{+}-\Delta-2 \gamma_{0} r \cos \left(K\left[z-z_{0}\right]\right)}{\xi_{+}+\Delta-2 \gamma_{0} r \cos \left(K\left[z-z_{0}\right]\right)}, \\
p & =\frac{2 r^{2} \cos ^{2}\left(K\left[z-z_{0}\right]\right)-2 \gamma_{0} r \cos \left(K\left[z-z_{0}\right]\right)+\xi_{-}}{\xi_{+}-2 \gamma_{0} r \cos \left(K\left[z-z_{0}\right]\right)}, \\
\eta_{0} & =\left(\xi_{+}-\Delta\right) /\left(\xi_{+}+\Delta\right),
\end{aligned}
$$

and the functions

$$
\begin{aligned}
& F_{ \pm}(\eta)=K\left(\sqrt{\frac{\xi_{-}-\Delta}{\xi_{-}+\Delta}}\right) \pm F\left(\sin ^{-1} \eta, \sqrt{\frac{\xi_{-}-\Delta}{\xi_{-}+\Delta}}\right) \\
& \Pi_{ \pm}(\eta)=\Pi\left(\eta_{0}^{2}, \sqrt{\frac{\xi_{-}-\Delta}{\xi_{-}+\Delta}}\right) \pm \Pi\left(\sin ^{-1} \eta, \eta_{0}^{2}, \sqrt{\frac{\xi_{-}-\Delta}{\xi_{-}+\Delta}}\right) \\
& S_{ \pm}(\eta)=\pi / 2 \pm \sin ^{-1} p
\end{aligned}
$$


where $K, F$ and $I$ are elliptic functions. The orbit is now

$$
t=\frac{N \pi}{\beta_{\mathrm{D}} K}+\frac{1}{K}\left\{\frac{2+\xi_{-}-\Delta}{\gamma_{0} \sqrt{2\left(\xi_{-}+\Delta\right)}} F_{ \pm}(\eta)+\frac{\Delta}{\gamma_{0}} \sqrt{\frac{2}{\xi_{-}+\Delta}} \Pi_{ \pm}(\eta)+\frac{1}{2} S_{ \pm}(p)\right\}
$$

with the plus sign for $z=2 N \pi$ to $z=(2 N+1) \pi$ and the minus sign for $z=(2 N+1) \pi$ to $z=2(N+1) \pi$ and where the integer, $N$, is the number of completed half periods. The drift velocity $\beta_{D}$, which is a constant of the particle motion, is given by

$$
\frac{1}{\beta_{\mathrm{D}}}=\frac{2}{\pi}\left[\frac{2+\xi_{-}-\Delta}{\gamma_{0} \sqrt{2\left(\xi_{-}+\Delta\right)}} K\left(\sqrt{\frac{\xi_{-}-\Delta}{\xi_{-}+\Delta}}\right)+\frac{\Delta}{\gamma_{0}} \sqrt{\frac{2}{\xi_{-}+\Delta}} \Pi\left(\eta_{0}^{2}, \sqrt{\frac{\xi_{-}-\Delta}{\xi_{-}+\Delta}}\right)\right] .
$$

The solution for trapped particles will not be written down here, it has only relatively minor differences from eq.(12). The parameter $\Delta$ has physical significance: it represents how close to trapping a particle is, being small (and real) for a nearly trapped particle. The limit for very small $\Delta$ can be treated directly (avoiding elliptic functions), however, we do not do this here.

\section{Single particle current}

The Fourier transform of the particle current (e.g. Melrose 1986, p.74) for our case $(\boldsymbol{x} \| \boldsymbol{v})$, as

$$
j(\omega, k)=q \int d z e^{i\left(\omega t(z)-k_{\|} z\right)},
$$

with $t$ given by eq.(12) with eq.(13). We can expand this current in two types of sum over harmonics. One leads to integral expressions for the contribution of each harmonic, the other (which we choose here) gives complicated expansions but results in analytic expressions. Expanding the orbit of an untrapped particle in harmonics of the longitudinal wave

$$
t=\sum_{p=1}^{\infty} C_{\mathrm{p}} \sin K p\left(z-z_{0}\right)+\frac{z}{\beta_{\mathrm{D}}}+\bar{t}
$$

where $\bar{t}$ is a constant and the coefficients $C_{\mathrm{p}}$ are the elliptic integrals

$$
C_{\mathrm{p}}=\frac{1}{p \pi K} \int_{0}^{2 \pi} \frac{m \gamma_{0} K-q E_{0} \cos z}{\sqrt{\left(m \gamma_{0} K-q \phi_{0} \cos z\right)^{2}-m^{2} K^{2}}} \cos p z d z
$$

yields (the current is now written as a scalar since its direction along $z$ is known)

$$
j(\omega, k)=q e^{i \omega t} \sum_{s=-\infty}^{\infty} e^{-i s z_{0}} V\left(s, \beta_{\mathrm{D}}, k\right) 2 \pi \delta\left[\omega-\beta_{\mathrm{D}}\left(k_{\|}-s K\right)\right]
$$

with

$$
V\left(\boldsymbol{s}, \beta_{\mathrm{D}}, \boldsymbol{k}\right)=\beta_{\mathrm{D}} \sum_{\zeta} J_{s-m(\zeta)}\left(\omega C_{1}\right) \prod_{p=2}^{\infty} J_{s_{\mathrm{p}}}\left(\omega C_{\mathrm{p}}\right)
$$

and

$$
m(\zeta)=\sum_{n=2}^{\infty} n s_{n},
$$

and where $\zeta$ is the countably infinite set of integers $\left\{8_{2}, \ldots, s_{n}, \ldots\right\}$. The sum in eq.(18) is thus over all possible choices of this set.

In order to perform meaningful calculations, an approximation of $V\left(s, \beta_{D}, k\right)$ is required. We note from the form of $C_{\mathrm{p}}$ given in eq.(16) that the first of the constants $(p=1)$ is largest, and thus we take all other $C_{\mathrm{p}}$ to be zero. In this approximation we retain in eq.(18) only contributions from Bessel functions with $C_{1}$ in their argument, or which are of zero order and so the only terms that remain in the sum over the set $\zeta$, are those with $s_{2}=s_{3}=\ldots=s_{n} \ldots=0$. Thus we have

$$
V\left(s, \beta_{\mathrm{D}}, \boldsymbol{k}\right) \simeq \beta_{\mathrm{D}} J_{s}\left(\omega C_{1}\right) \text {. }
$$


The constant $C_{1}$ has been obtained analytically from eq.(16) (as can all $C_{\mathrm{p}}$ ) using an approximation which does not involve elliptic integrals. It involves an alternative calculation of the particle orbit by expanding the Hamiltonian in the rest frame, eq.(1), in the electric field. When carried to all orders, it gives exact summation forms for $C_{\mathrm{p}}$. One finds that for untrapped particles (cf. Rowe 1989)

$$
C_{1} \simeq\left(q E_{0}\right) /\left(m \gamma_{0}^{3} v_{0}^{3} K^{-2}\right) .
$$

A final point to note here is that for a truly useful result the constant $v_{0}$ (the zero order particle velocity) needs to be expressed in terms of the drift velocity, $\beta_{\mathrm{D}}$, which is the true constant of the motion. One cannot invert analytically the definition of $\beta_{\mathrm{D}}$ given by eq.(13), however, if the expansion method used to approximate $C_{1}$ is applied here, one finds that the drift velocity is equivalent to $v_{0}$ up to second order in $E_{0}$. The second order correction to $\beta_{\mathrm{D}}$ results in a quintic equation for $v_{0}$, and consequently we shall assume for our purposes that $\beta_{\mathrm{D}}$ and $v_{0}$ are the same.

\section{Propagating waves}

To extend the previous results to a propagating electrostatic wave one can Lorentz transform the particle current and write all rest frame quantities in terms of quantities in the new frame of reference. Fourier transforming Maxwell's equations implies (e.g. Melrose 1986, p.10)

$$
\rho(\omega, \boldsymbol{k})=k_{\|} j(\omega, \boldsymbol{k}) / \omega,
$$

and a Lorentz transform, with Lorentz factor $\gamma_{\mathrm{R}}$, along the direction of motion of the particle and electrostatic wave results in

$$
\begin{aligned}
j^{\prime}(\omega, \boldsymbol{k}) & =\gamma_{\mathrm{R}}\left[j(\omega, \boldsymbol{k})-v_{\mathrm{R}} \rho(\omega, \boldsymbol{k})\right] \\
& =\frac{\omega^{\prime}}{\omega} j(\omega, \boldsymbol{k})
\end{aligned}
$$

with other quantities transforming in the usual way. One can write down the quantities given in the previous section in terms of the new frame parameters but here we will give only the new frame version of $C_{1}$ corresponding to eq.(21):

$$
\gamma_{\phi} C(1) \simeq \frac{\psi^{\prime} v_{\phi}^{2}}{\gamma_{0}^{\prime 3}\left(v_{0}^{\prime}-v_{\phi}\right)^{3} \Omega^{\prime}}
$$

where

$$
\psi^{\prime}=\left(q E_{0}^{\prime}\right) /\left(m \Omega^{\prime}\right)
$$

is a dimensionless quantity in our units, $v_{\phi}$ is the phase speed of the longitudinal wave and $\gamma_{\phi}$ the corresponding $\gamma$ factor. The current in the new frame is

$$
j(\omega, \boldsymbol{k})=q e^{i \phi^{\prime}} \sum_{s=-\infty}^{\infty} e^{-i s \alpha^{\prime}} V^{\prime}\left(s, \beta_{\mathrm{D}}^{\prime}, \boldsymbol{k}^{\prime}\right) 2 \pi \delta\left[\omega-s \Omega-\beta_{\mathrm{D}}\left(k_{\|}-s K\right)\right],
$$

with

$$
V^{\prime}\left(s, \beta_{\mathrm{D}}^{\prime}, \boldsymbol{k}^{\prime}\right)=\frac{\beta_{\mathrm{D}}^{\prime}-v_{\phi}}{1-n^{\sigma} v_{\phi} \cos \theta} \sum_{\zeta} J_{s-m(\zeta)}\left(\left[\omega^{\prime}-v_{\phi} k_{\|}^{\prime}\right] \gamma_{\phi} C_{1}\right) \prod_{p=2}^{\infty} J_{s_{\mathrm{p}}}\left(\left[\omega^{\prime}-v_{\phi} k_{\|}^{\prime}\right] \gamma_{\phi} C_{\mathrm{p}}\right),
$$

where $n^{\sigma}$ is the refractive index for a given mode of radiation, $\sigma$, and $\theta$ is the angle of emission to the magnetic field. The current given above is valid for untrapped particles accelerated by a longitudinal wave of arbitrary phase speed.

\section{Absorption coefficient}

The current given above can be used to write down the absorption coefficient for waves emitted by linearly accelerated particles, which at a given harmonic is defined generally as (e.g. Melrose 1980, Ch.5)

$$
\gamma_{s}^{\sigma}(k)=-\int d^{3} p_{\mathrm{D}} \frac{P_{s}^{\sigma}(k)}{\hbar \omega^{\sigma}} \Delta p_{\mathrm{D}} \cdot \frac{\partial F\left(p_{\mathrm{D}}\right)}{\partial p_{\mathrm{D}}}
$$


where

$$
P_{s}^{\sigma}(\boldsymbol{k})=8 \pi^{2} q^{2} R_{E}^{\sigma}(\boldsymbol{k})\left|e^{\sigma}(\boldsymbol{k}) \cdot j_{s}(\omega, \boldsymbol{k})\right|^{2},
$$

is the power emitted by a current $j,(\omega, k)$ at a given harmonic in a mode of radiation, $\sigma$. The ratio of electric to magnetic energy in the wave mode is

$$
R_{E}^{\sigma}(\boldsymbol{k})=\left(2 n^{\sigma} \partial\left[\omega n^{\sigma}\right] / \partial \omega\right)^{-1},
$$

for transverse waves in an isotropic, homogeneous medium (this will not apply in a 'real' pulsar but we shall assume it here) and the polarization vector is $e^{\sigma}(k)$. In eq.(28), one identifies $p_{D}$ as the drift momentum, $p_{\mathrm{D}}=m \boldsymbol{\beta}_{\mathrm{D}} \gamma_{\mathrm{D}}$, a constant of the particle motion. One can determine $\Delta p_{\mathrm{D}}$, the change in the drift momentum on an emission, by appealing to the resonance condition for a given harmonic embodied in the delta functions in the current. For a specific harmonic one finds

$$
\Delta p_{\mathrm{D}}=k_{\|}-s K \text {. }
$$

A simplifying assumption is to choose a one dimensional distribution function, $F\left(p_{\mathrm{D}}\right)=f\left(p_{\mathrm{D} \|}\right) \delta\left(p_{\perp}\right)$, which is consistent with our treatment of particles moving along the magnetic field. A one-dimensional distribution is also consistent with a pulsar magnetosphere where, due to the high magnetic fields (up to $10^{12} \mathrm{G}$ ), particles rapidly loose perpendicular momentum. We split the growth rate into two parts

$$
\gamma_{\boldsymbol{s}}^{\sigma}(\boldsymbol{k})=-\int d p_{\mathrm{D} \|} \frac{P_{\boldsymbol{s}}^{\sigma}(\boldsymbol{k})}{\hbar \omega^{\sigma}} \Delta p_{\mathrm{D} \|} \frac{\partial f\left(p_{\mathrm{D} \|}\right)}{\partial p_{\mathrm{D} \|}}+\int d p_{\mathrm{D} \|} \frac{\partial}{\partial p_{\mathrm{D} \perp}}\left[\frac{P_{\boldsymbol{s}}^{\sigma}(\boldsymbol{k})}{\hbar \omega^{\sigma}} \Delta p_{\mathrm{D} \perp}\right]_{p_{\mathrm{D} \perp}=0} f\left(p_{\mathrm{D} \|}\right)
$$

the second of which is zero for this mechanism.

Using eqs.(29), (31) and (32) and setting $\omega=a \Omega$, the absorption coefficient reduces to (dropping primes in the new reference frame)

$$
\begin{aligned}
\gamma_{s}^{\sigma}(k) \simeq & \left.-\frac{4 \pi^{2} q^{2} m \cos ^{2} \phi \sin ^{2} \theta a\left(n^{\sigma} a v_{\phi} \cos \theta-s\right) v_{\phi}^{2}}{n^{\sigma} \partial\left[a n^{\sigma}\right] / \partial a \Omega\left\{\left(a v_{\phi} n^{\sigma} \cos \theta-s\right)^{2}-v_{\phi}^{2}(a-s)^{2}\right\}^{3 / 2}}\right)\left.\right|^{2} \\
& \mid J_{s}\left(\psi \frac{\left\{\left(a v_{\phi} n^{\sigma} \cos \theta-s\right)^{2}-v_{\phi}^{2}(a-s)^{2}\right\}^{3 / 2}}{a^{2} v_{\phi}\left(1-n^{\sigma} v_{\phi} \cos \theta\right)^{2}}\right), \\
& \frac{\partial f}{\partial p_{\mathrm{D} \|}}\left(\frac{m v_{\phi}(a-s)}{\left\{\left(a v_{\phi} n^{\sigma} \cos \theta-s\right)^{2}-v_{\phi}^{2}(a-s)^{2}\right\}^{1 / 2}}\right),
\end{aligned}
$$

where we have used the approximate form of $C_{1}$ and again retained only the first Bessel function in our current expansion. The angle $\phi$ is the polarization angle with respect to the plane of the magnetic field and $k$. It was noted in the introduction that a one-dimensional, relativistic plasma will support (if not prefer) superluminal oscillation modes. This point should be investigated in detail for plasmas with specific distribution functions relevant to pulsar magnetospheres, as should the question of generation of such waves. We do not do this here, rather the purpose of this section is to assume that superluminal longitudinal waves are present in pulsar magnetospheres and to discuss the consequences for the linear acceleration emission maser.

We note firstly that the above calculations are valid for superluminal waves, $\Omega>K$ in our units, regardless of the fact that we started in a wave rest frame (one could in principle derive the results for arbitrary $\Omega$ and $K$ from the second order equation of particle motion). Secondly, our calculations have been for untrapped particles which is the only scenario possible for this case, and finally emission of longitudinal waves can also be treated, however we concentrate on transverse waves which are the directly observable quantities.

The first suggestion that superluminal waves admit unique effects is in the resonance condition describing emission at the $s^{\text {th }}$ harmonic by a particle accelerated by a longitudinal wave,

$$
\beta_{\mathrm{D}}=\frac{v_{\phi}(a-s)}{n^{\sigma} a v \phi \cos \theta-s}
$$

If the denominator is zero we require also that the numerator be zero. This occurs on the cone

$$
\cos \theta_{c}=\left(n^{\sigma} v_{\phi}\right)^{-1},
$$

and at the frequency $\omega=s \Omega$ [the result for the growth rate can be shown to reduce to that of Melrose $(1978)$ in the limit $K \rightarrow 0$, in which case $\cos \theta_{c} \rightarrow \infty$ ]. We note the following points: 
i. only if the longitudinal waves are superluminal $\left(\left|v_{\phi}\right|>\left|v_{\omega}\right| \sim 1\right.$, where $v_{\omega}$ is the phase speed of the emitted wave) is $\theta_{c}$ a real angle;

ii. particles with positive drift velocity emit at $\omega>s \Omega$ inside the cone and at $\omega<s \Omega$ outside the cone. The reverse is true for particles with negative drift velocity;

iii. for $\theta_{c}$ to be an observable quantity (in some sense) it must be small (i.e. an inside to outside cone transition must occur within a narrow field of observation) and thus $v_{\phi}$ is positive $\sim c$.

Choosing the numerator and denominator of eq.(34) to be zero does not guarantee resonant particles there, in fact if we consider instead the condition $\left|\beta_{\mathrm{D}}\right|<1$, we find that within the band

$$
\cos \theta_{\mathrm{c}}\left[\frac{s}{a}-v_{\phi}\left|1-\frac{s}{a}\right|\right]<\cos \theta<\cos \theta_{\mathrm{c}}\left[\frac{s}{a}+v_{\phi}\left|1-\frac{s}{a}\right|\right],
$$

about the cone angle no resonance is possible. The angle $\theta_{c}$ is then a point of demarcation between two types of emission, in separate frequency ranges and at different angles.

The absorption coefficient eq.(33), contains a factor, $\left(n^{\sigma} a v_{\phi} \cos \theta-s\right)$, which is zero when $\cos \theta=$ $s /\left(n^{\sigma} a v_{\phi}\right)$, and thus the absorption changes sign within the forbidden region. Table 1 gives the require-

Table 1 The possibilities for maser amplification of the radiation emitted by linearly accelerated charged particles in the field of a superluminal electrostatic wave

\begin{tabular}{|c|c|}
\hline$\theta<\theta_{\mathrm{c}}$ & $\theta>\theta_{\mathrm{c}}$ \\
\hline$\frac{\partial f}{\partial p_{\mathrm{D} \|}}>0$ & $\frac{\partial f}{\partial p_{\mathrm{D} \|}}<0$ \\
\hline
\end{tabular}

ment on the distribution function for amplification to occur. It shows that amplification of the radiation is possible in both emission regions, due to different particles in the distribution. A general analysis of the absorption coefficient at the first harmonic reveals that for a given frequency the emission peaks near the field lines, drops away in the forbidden region and then peaks again before falling to zero. Over a large frequency range (several times $\Omega$ ) above $\Omega$, emission is due to fast particles and is confined to small angles from the magnetic field. Emission below $\Omega$ is spread more widely over angle and is confined to a narrower frequency range. The angle $\theta_{c}$ can be made as small as required by taking $v_{\phi}$ as close to $c=1$ as is necessary.

A physical understanding of the table is based on the equation of conservation of energy in the particles and waves. The equation can be derived directly from the quasilinear equations describing the evolution of both wave and particle distributions (e.g. Melrose 1980, p.160). In the present case we find for each mode of radiation:

$$
\frac{d}{d t}\left[\int d p_{\mathrm{D} \|} \epsilon_{\mathrm{D}} f\left(p_{\mathrm{D} \|}\right)+\sum_{s} \int \frac{d^{3} \boldsymbol{k}}{(2 \pi)^{3}} \hbar(\omega-s \Omega) N_{s}^{\sigma}(\boldsymbol{k})\right]=0,
$$

where $N_{s}^{\sigma}(k)$ is the number density of photons in mode $\sigma$ due to emission at the $s^{\text {th }}$ harmonic. The first term describes the rate of energy change in the particles, the second in the emitted waves and the last in the longitudinal waves. This last term shows that the process can be viewed as a sum of all order linear scatterings of the longitudinal waves by particles into transverse waves; taken in full we have a non-linear process. Emitted waves are amplified and the longitudinal waves damped if $d N_{a}^{\sigma}(k) / d t$ is positive. For frequencies $\omega>s \Omega$, at the $s^{\text {th }}$ harmonic, energy conservation requires that the particles near the resonance lose energy. This occurs if there are more above the resonance energy than below it (a rising distribution for positive velocities and a falling one for negative velocities). In this case, both particles and longitudinal waves lose energy to the transverse waves. For $\omega<s \Omega$, transverse waves are amplified if the particles also gain energy and this occurs if more particles lie below the resonance energy than above it (a falling distribution for positive velocities and a rising one for negative velocities). In this case, both particles and transverse waves gain energy from the longitudinal waves. 


\section{Application to pulsars}

One class of radio pulsars are those with multiple components. The triple component pulsars have been taken as a prototype for a phenomenological model of pulsars by Rankin (1983a) and others, and several have been scrutinized individually, including the frequency evolution of their average pulse profiles (e.g. Hankins and Rickett 1986). As discussed in these papers it appears that there are two types of emission from radio pulsars: core emission appearing in the center of profiles and conal emission appearing on the outside edges. The following points are important:

i. the appearance of distinct pulse components with emission falling off between them, and

ii. the central core component appears to dominate the profiles at lower frequencies while the conal component dominates at higher frequencies.

Phenomenologically, the distinct emission components are taken to represent two concentric annular emission regions about a central core emission region. It is plausible that these different regions are actually manifestations of angular structure in the emission mechanism itself, not physically distinct regions of the magnetosphere. One appealing feature of this hypothesis is that one does not have to explain how distinct emitting regions about the pole are to be created-we have a single emitting region and a single mechanism seen from different angles.

We do not attempt to construct a complete model here, this has yet to be done, however, a sketch of a model is as follows. Consider field lines coming from near the polar cap. The precise nature of the field lines in these regions is unknown, however linear acceleration emission favors straight field lines. If sparking across a polar gap, or some other mechanism, can be found to generate superluminal waves then such waves would propagate along the field lines causing particles to radiate. Suppose the waves propagate only on or within a narrow cone about the polar cap. In that case the absorption coefficient has an angular dependence about each field line on the cone, the total observed emission being the sum of emission along our line of sight from each part. Each point of the cone will contribute different amounts to the emission depending on the angle of our line of sight to the field at that point and, throughout a pulse different parts of the cone will dcminate the emission, giving it a distinct linear polarization $(<100 \%)$ which will rotate throughout the pulse. The important questions are

i. can the angular behavior of the growth rate about a single field line recreate the pulsar profiles with multiple components when integrated over a cone, and

ii. how can superluminal waves be generated and amplified?

\section{Conclusion}

We have given more detailed calculations for linear acceleration emission than are to be found elsewhere, the most important of which is the growth rate. We have shown that in the case of superluminal waves an angular variation in the growth occurs which implies two separate angular regions of emission favoring different frequency regimes roughly separated at $\Omega$ (this can be taken to be the plasma frequency). We have suggested that the angular variation may be used to explain the multicomponent pulsar pulse profiles and this application is presently under consideration. 